\section{Principles and Practice of Clinical Research}

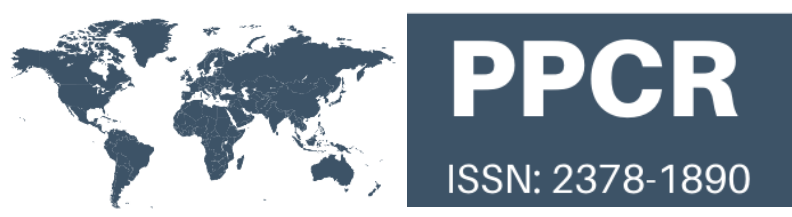

\title{
Combination of olanzapine with bispectral index guided anesthesia to prevent postoperative delirium in elderly patients undergoing major gastrointestinal elective surgery: a randomized, double-blind, placebo-controlled study protocol.
}

\author{
R. Quimas Molina da Costa ${ }^{1}$, O. Zied Alzobi ${ }^{2}$, S. Al-Busaidi ${ }^{3}$, D. Arbaje ${ }^{4}$, C. Avery Lavender ${ }^{5}$, V. \\ Andrade Batistella ${ }^{6}$, E. Boschetti Grutzmacher ${ }^{7}$, G. Casais ${ }^{8}$, D. Castro ${ }^{9}$, M. Cohen ${ }^{10}$, A. García \\ Delgado $^{11}$, M. Haseeb ${ }^{12}$, V. Hernandez Leon ${ }^{13}$, P. Hilton de Andrade Filho ${ }^{14}$, B. Khouri Lopez ${ }^{15}$, A. \\ Marcadenti ${ }^{16}$, J. Müller ${ }^{17}$, D. Noya Brandao ${ }^{18}$, F. Viecili Tarcha ${ }^{19}$, J. Tepedino Martins Alves ${ }^{20}$, F. Urrutia \\ Goldsack $^{21}$, E. Youssif ${ }^{22}$ A. Tamayo ${ }^{23}$, DS. Schwartz ${ }^{24}$, D. Garcia Choza ${ }^{25}$, M. Cubria ${ }^{26}$, I. Sulieman ${ }^{27}$ \\ \#All authors contributed equally \\ *Corresponding author: Raquel Quimas Molina da Costa. R. Diniz Cordeiro 30, 3rd floor. Botafogo, Rio de Janeiro - RJ. 22281-100, Brazil. Email: \\ raquel.costa@idor.org \\ Rest of author's affiliation at the end of the manuscript.
}

Received May 27, 2021; accepted September 15, 2021; published October 14, 2021.

\begin{abstract}
:
Introduction: Postoperative delirium (POD) is an important complication of major surgery in elderly patients. It increases morbidity and mortality, hospital stay, and total healthcare costs. Since no treatment has proven effective once POD is established, prevention is key. Evidence exists that bispectral index guided anesthesia (BIS-GA) and antipsychotics may independently reduce incidence of $P O D$, but the efficacy of combining these preventive strategies is unknown. Objective: To compare the combination of olanzapine + BIS-GA with BIS-GA alone for prevention of POD in elderly patients undergoing major elective surgery.

Methods: We propose a Phase II, multi-center, randomized, double-blind, parallel, placebo-controlled trial. The study arms will be BIS-GA + two doses of olanzapine $5 \mathrm{mg}$ given pre and postoperatively compared with BIS-GA + placebo in patients $\geq 65$ years hospitalized for major elective surgery. Exclusion criteria include cardiac-and neurosurgery, dementia history, concurrent antipsychotic, anticholinergic, or sedative-hypnotic use, olanzapine allergy, delirium at hospital admission, cognitive impairment and inability to be interviewed. The primary outcome is incidence of POD diagnosed by DSM-V criteria and assessed by the Confusion Assessment Method (CAM) scale. Secondary outcomes include delirium severity, rescue therapy use, length of hospital stay and incidence of adverse events.

Discussion: There is an increasing need for trials that advance knowledge in prophylactic methods to prevent delirium. By combining two preventive methods, we expect to decrease the incidence of POD, which will result in decreased morbidity, mortality, and total healthcare costs.
\end{abstract}

Keywords: Delirium; Postoperative Complications; Consciousness Monitors; Olanzapine; Randomized Controlled Trial [Publication Type].

DOI: http://dx.doi.org/10.21801/ppcrj.2021.73.5 


Abbreviations:
BIS-GA: bispectral index guided anesthesia
CAM: Confusion Assessment Method
CAM-S-lf: Confusion Assessment Method - Severity -
long-form
CIs: 95\% confidence intervals
CRF: case report form
DSM-V: Diagnostic and Statistical Manual of Mental
Disorders, fifth edition
ECG: Electrocardiogram
HRs: hazard ratios
POD: postoperative delirium
RCT: Randomized Clinical Trial
TMG: Trial Management Group

\section{INTRODUCTION}

Delirium, an acute disturbance in attention and awareness that occurs over hours to days consequent to a medical condition/procedure, is characterized by inattention, fluctuating consciousness levels, and cognitive impairment (APA, 2013) and is associated with neurotransmitter imbalance caused by irregular response of tissues to normal levels of stress (MacLullich et al., 2008). POD is an important complication that affects mostly elderly patients after major surgical procedures (Whitlock et al., 2011), with prevalence up to $65 \%$ (Maldonado et al., 2008). It increases morbidity and mortality (Witlox et al.,2010), length of hospital stay (Gleason et al., 2015), and total healthcare costs (Leslie et al., 2008). It is challenging to treat and even after treatment, patients can have persistent functional impairment, increased rates of institutionalization, and increased dementia rates (Witlox et al., 2010). In contrast to Postoperative Cognitive Dysfunction (PCD), which is identified through neuropsychological testing and persists for several years after surgery, POD is usually observed clinically with a fluctuating course, and is related to hospitalization (Evered et al., 2018).

Since at least $30-40 \%$ of delirium cases could be prevented (Gonzalvo et al., 2017), successful prevention strategies could significantly decrease the healthcare system burden (Leslie et al., 2008). Nonpharmacological approaches such as mobilization, therapeutic activities, hydration, proper nutrition, sleep strategies, hearing/vision adaptations (Hshieh et al., 2015), and BIS-GA (Chan et al., 2013; Siddiqi et al., 2016) have been used to effectively decrease the incidence of POD. Brain function monitoring using BIS-GA is considered an effective non-pharmacological method to reduce POD and promote early recovery (Chan et al., 2013; Lewis et al., 2020).

Evidence for the efficacy and tolerability of pharmacological treatment is limited (Rivière et al., 2019). Olanzapine is a second-generation antipsychotic that may effectively treat delirium, providing sedation without significant extrapyramidal side effects (Markowitz et al., 2008). A randomized clinical trial conducted in elderly patients after joint-replacement surgery showed a significantly lower incidence of POD with prophylactic olanzapine (Lanser et al., 2010). However, there is no evidence supporting the use of olanzapine in addition to a non-pharmacological intervention such as BIS-GA for delirium prevention in elderly patients after elective surgeries.

We hypothesize that prophylactic olanzapine would reduce POD incidence among patients $\geq 65$ years old who have undergone major gastrointestinal elective surgery using BIS-GA. Our purpose is to evaluate the incidence of delirium in the treatment group (olanzapine + BIS-GA) compared to placebo (BIS-GA alone). As secondary objectives, we will examine the delirium severity, rescue therapy use, side effects incidence, and the length of hospital stay in both groups.

\section{MATERIALS AND METHODS}

\section{Trial Design}

This study will be a phase II, multi-center, randomized, double-blind, placebo-controlled trial.

\section{Study Setting}

This study will be conducted in tertiary hospitals, where BIS-GA is used as a standard of care for major elective surgeries.

\section{Randomization}

All eligible patients who consent to the study participation will be randomly assigned to one of the two treatment arms: Olanzapine or matching placebo with 1:1 allocation ratio. The randomization sequence will be generated by a biostatistician using a computerized random number generator. Blocked randomization using variable block sizes of four and eight individuals will be implemented to ensure balance between groups and to avoid guessing of the randomization sequence.

Once eligibility criteria are confirmed and the informed consent form signed, the investigator will enter data in the system and a randomization number 
will be generated. The study pharmacist will verify the randomization number assigned to the patient as a user of the same system. He/she will not have any interaction with the patients and will deliver an indistinguishable package (olanzapine/matching placebo) to the responsible nurse.

\section{Blinding}

The study will be double-blinded. Patients, outcome assessors, and healthcare providers (surgeons, anesthesiologists, clinicians, and nurses) responsible for the patient care pre and post-operatively will remain blinded upon study completion.

Guarantee of blinding will be achieved by keeping the randomization data strictly confidential until the time of unblinding (if necessary) or until the end of the study. Only the unblinded team, composed of the study pharmacist (in charge of treatment allocation), the principal investigator, and one external physician, will know these data. In addition, the identity of the treatments will be concealed by the use of placebo pills that will be identical in packaging, labeling, administration time, appearance (color and size) and odor as the active agent.

\section{Emergency unblinding}

Emergency code breaks must only be undertaken when required for patient safety. If knowledge of assigned treatment is essential for management of the patient in a critical situation, code breaks may be requested to the unblinded team. The investigator will receive details of the patient's assigned treatment and will share this information with the patient's healthcare providers. Treatment allocation will also be revealed to the patient if unblinding is necessary. It is mandatory for the investigator to record the code break and the justification for it. Unblinded participants will be kept in the final analysis, unless consent is withdrawn.

\section{Eligibility Criteria}

Eligibility will be determined from medical records when the surgery is scheduled or from patients' interviews at the time of hospital admission.

Inclusion criteria: patient $\geq 65$ years who will undergo major gastrointestinal elective surgery with BIS-GA (either upper or lower gastrointestinal, laparoscopic or open abdominal, oncologic or nononcologic surgeries) and able to give informed consent.

Exclusion criteria: expected impossibility of enteral feeding within 24 hours postoperatively; dementia history; concurrent use of antipsychotic, anticholinergic, or sedative-hypnotic medications; olanzapine allergy; morphine allergy; delirium at hospital admission and history/evidence of cognitive impairment [measured by the Montreal Cognitive Assessment (MoCA) score of 25 or less (Nasreddine et al., 2005)].

\section{Recruitment Strategy}

We expect a two-year recruitment period. Advertising directed to healthcare providers of various surgical subspecialties from the study sites will encourage referrals of potential study participants. All patients admitted for major gastrointestinal elective surgery will be considered. (Figure 1). Potential participants will be screened for eligibility criteria when surgery is scheduled.

\section{Adherence}

We expect a high level of adherence since patients will be hospitalized. The designated study nurse will be responsible for the administration of the assigned treatment, documenting the treatment in the patient's medical record.

\section{Interventions}

Randomized patients will receive either two doses of olanzapine $5 \mathrm{mg}$ tablets or matched placebo in a 1:1 ratio according to their allocation group. The 1st dose will be administered two hours before surgery and the 2nd within 24 hours after surgery, as soon as the patient is able to tolerate oral or enteral feeding.

This dosing regimen showed efficacy in reducing the occurrence of POD in a previous study (Larsen et al., 2010). Furthermore, the dosage of $5 \mathrm{mg}$ daily is effectively and safely used for short-term treatment of delirium in critically ill patients (Skrobik et al., 2004). Olanzapine reaches its maximum plasma concentration within 6 hours after oral administration and has half-life ranges from 21-54 hours (5th to 95th percentile; mean 30 hours). The incidence of POD is highest in the first 4 days after surgery (Litaker et al., 2001). Therefore, the long half-life of olanzapine would ensure plasma levels across the expected time of high incidence of delirium.

All patients will receive BIS-GA as standard of care during the procedure, with the index target value of 4060 and suppression rate of 0 , which has demonstrated to be effective in POD prevention (Chan et. al. 2013).

All patients will be admitted in the ICU as standard of care after major surgical procedures. In order to 


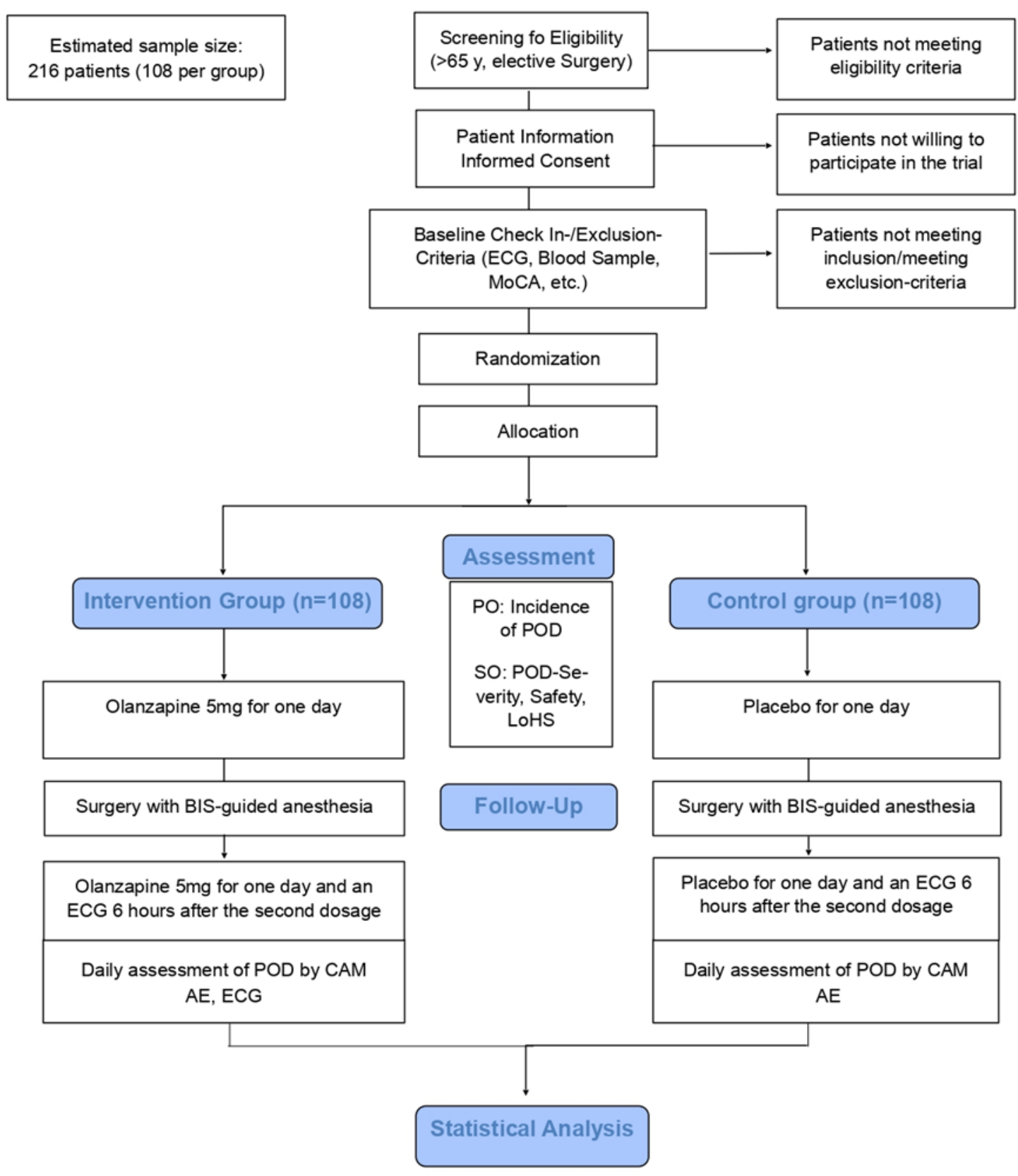

Figure 1.CONSORT flow diagram. AE: adverse events; BIS: bispectral index; CAM: Confusion Assessment Method; ECG: electrocardiogram; LoHS: length of hospital stay; MoCA, Montreal Cognitive Assessment: PO: primary outcome; POD: postoperative delirium; SO: secondary outcomes.

standardize postoperative pain management, which could also influence POD, an intravenous morphine patient-controlled analgesia (PCA) device will be used for all patients. They will receive a $2 \mathrm{mg}$ bolus dose of morphine for each request with a 5 min lockout period and a safe higher limit of three boluses per hour.

\section{Modification/discontinuation}

For patients who cannot tolerate oral medications, but can receive enteral feeding, orally disintegrating tablets of olanzapine or the matched placebo will be used. In a case of post-surgical complications that prohibit oral or enteral feeding by 24 hours (second dose), the patient outcomes will be followed for data record. Patients who develop any hypersensitivity reaction to the first administration will not receive the second dose.

\section{Outcomes}

The primary outcome will be the incidence of POD, which will be diagnosed by CAM scoring system and 
evaluated within 6-24 hours after surgery. Additional evaluations will take place every 24 hours until hospital discharge or up to 7 days after surgery. The CAM is a widely recognized tool for the screening and diagnosis of delirium (Inouye et al., 1990), and it considers the Diagnostic and Statistical Manual of Mental Disorders, fifth edition (DSM-V) criteria as well as delirium's fluctuating nature. By using four criteria (acute onset/fluctuating course; inattention; disorganized thinking; altered level of consciousness) with dichotomous responses (yes $=1 / \mathrm{no}=0$ ), it allows an accurate diagnosis within 3-5 minutes with 94\%-100\% sensitivity and 90\%-95\% specificity (Inouye et al., 1990). The presence of 3 or 4 criteria, reported as binary outcomes (present/absent), at least once during the observed period indicates delirium. We will use the CAM version validated in Brazilian Portuguese (Fabbri et al., 2001).

Secondary outcomes include: 1) Delirium severity [assessed every 24 hours using CAM- Severity longform (CAM-S-lf), with score range 0-19 (19=most severe)]; 2) Delirium duration (in days); 3) Safety parameters including QTc intervals assessed by ECG (at baseline, 6 hours after the second dose and every 24 hours thereafter), extrapyramidal symptoms (assessed by staff member or by self-reporting) using the Simpson-Angus Scale (Knol et al., 2009), and any potential adverse events described in the approved label of olanzapine and graded by the Common Terminology Criteria for Adverse Events (Trotti et al., 2003); 4) Rescue therapy use (total milligrams of additional antipsychotics); 5) length of hospital stay (evaluated retrospectively in days); and 6) Delirium time of onset (in days).

\section{Data Collection and Management}

A Case Report Form (CRF) previously approved by the ethics committee will be used to collect all trial data. These forms will be completed by the investigators and outcome assessors, based on information from patients' medical records. Parameters to be extracted from medical records include primary and secondary outcomes, demographics (age, gender, ethnicity, and marital status), and perioperative information (concomitant medications including total opioid consumption, anesthetic technique, intraoperative fluid records, blood transfusion and total surgical time).

The CRFs will include the participant trial ID with the study code (BISOLAD-0001, BISOLAD-0002, sequentially) and will be stored in a secure location. In a distinct secure location, an identification log will record the patient's name and study code.

The principal investigator will establish a Trial Management Group (TMG) (Harman et al., 2015) formed by physicians, nurses, a site coordinator, informatics technician, and pharmacist. Only healthcare professionals that assist patients directly, outcome assessors and the site coordinator will know the participant trial ID. The pharmacist will keep a registry of the intervention received by each code, and only the pharmacist will handle this information.

The informatics technician will create and manage access to a secure database to upload the CRF data. The database will be scheduled to back up every two weeks. It is the site coordinator's responsibility to upload and update the trial data weekly. The principal investigator will assign another member of the TMG to review the database weekly and assess inconsistencies and incomplete data.

\section{Interim Analysis}

Due to the small sample size and the moderate expected effect size, we will not adopt an interim analysis for efficacy.

\section{Sample Size Calculation}

Based on previous studies, we expect an 18\% POD incidence in the control group (Chan et. al., 2013; Zhou et. al., 2018) and a 5\% incidence in the intervention group (Larsen et al., 2010). Considering a 0.05 twosided significance level and $80 \%$ power, a total of 204 participants were calculated. An additional 5\% was calculated due to potential loss to follow-up, resulting in 216 participants or 108 per group.

\section{Statistical Analysis}

Continuous variables will be presented as means and standard deviations or medians and interquartile range depending on the data distribution. Categorical variables will be presented as relative and absolute frequencies. The normality of the variables will be assessed by the Kolmogorov-Smirnov test, skewness and kurtosis, and by visual inspection of the histogram.

The POD occurrence in both arms will be defined at the end of follow-up. Fisher's exact test will be used for comparing the POD incidence between treatment groups. As a secondary analysis, we will use the KaplanMeier survival method and log-rank test to compare time-to-event between olanzapine and placebo groups. Hazard ratios (HRs) with respective 95\% confidence 
intervals (CIs) will be described, and the influence of baseline characteristics over the POD incidence until discharge or up to 7 days (whichever comes first) will be evaluated using the Cox Regression test. Schoenfeld residuals will be used to check the proportional hazards assumption, and nonlinearity will be assessed using Martingale residuals. Adjustments will be made only if important baseline characteristics are unbalanced.

Other secondary outcomes will be evaluated as follows: 1) The CAM-S-lf scores will be divided in tertiles and compared between groups using Fisher's exact test; 2) Safety outcomes will be dichotomized (presence/absence) and will also be compared using the Fisher's exact test; 3) Delirium duration and length of hospital stay will be compared between groups using the Student's t-test or Mann-Whitney test; and 4) The rescue therapy use will be described in $\mathrm{mg} /$ day and compared between groups using Student's t-test or the Mann-Whitney test. In addition, sensitivity analysis will be performed between arms only with patients who complete the protocol to verify if the anesthetic technique, intraoperative imbalance, blood transfusion, use of different medications during anesthesia, total ICU time, or total opioid consumption affected results. If any of these variables have influenced the results, they will be adjusted for.

Analyses will be conducted according to the intention to treat principles and will consider a twotailed alpha of $5 \%$. STATA® version $16 \mathrm{C}$ program (StataCorp LLC) will be used to perform all statistical tests.

\section{Missing Data}

Missing data will be handled using multiple imputation techniques. Additional per-protocol analysis will be conducted excluding patients who could not take the second pill and those who have been unblinded. The amount of missing data for each variable will be described through proportion.

\section{DISCUSSION}

We propose a phase II, multicenter, double-blinded, randomized, placebo-controlled, parallel-group trial, to investigate the efficacy and safety of olanzapine in elderly patients undergoing major gastrointestinal elective surgery using BIS-GA.

POD is a very common source of morbidity in the postoperative period of major surgeries, particularly in elderly patients. Previous studies have shown efficacy for both pharmacologic and nonpharmacologic treatments in the prevention of POD, but these have not been studied in combination. This proposed trial would expand knowledge in prophylactic methods to prevent POD, resulting in improved outcomes and reduced healthcare costs.

Strengths of our study include the simple dosing protocol, a sample of inpatients that allow for higher adherence, the use of BIS-GA in both groups that eliminates confounding effects, and the homogeneity of the surgery type. Combining olanzapine with the use of BIS-GA, an effective POD prevention method consistent with current best practices, makes this research question relevant and innovative. Other relevant aspects such as safety parameters, the need for rescue therapy, or delirium time of onset are included as secondary outcomes and address important clinical implications and directions for future studies. For example, a delay in time to develop delirium could be explained by olanzapine concentrations in plasma, which would indicate the need for additional olanzapine dosing.

We recognize the limitations of the short follow-up time, which may not identify delayed presentation of POD, and the use of an oral study drug, which consequently eliminates patients with delayed oral or enteral feeding after surgery. Additionally, since we expect lower rates of POD in patients undergoing surgery with BIS-GA, a smaller difference between groups may occur, biasing results towards a negative outcome.

One main point of concern is how intraoperative complications may affect POD incidence. The randomization process and blinding of the anesthesiologist account for possible imbalance between groups should surgical complications occur. However, this may result in confounding factors that could influence the understanding of the study results. Another important condition is the emergence of PCD, a significant postoperative outcome in elderly people. Deficits in attention levels that characterize PCD may mimic POD. The later PCD occurrence and the nonfluctuating course of presentation help differentiate these conditions (Evered et. al., 2018). This study does not investigate the PCD incidence or severity, and this remains a research question for future studies.

\section{CONCLUSION}

The present study protocol gives important considerations regarding POD prevention. It takes into account the current knowledge of POD's major risk factors, management, and differential diagnosis, and therefore presents a feasible and valid design to help 
advance the field of treatment for adverse surgical outcomes.

\section{Acknowledgements}

This protocol was the result of a multidisciplinary group project for the Principles and Practice of Clinical Research (PPCR) course in 2020. The authors would like to thank all Teaching Assistants for the support during this project; Professor Felipe Fregni for the learning and opportunity; and the PPCR faculty team for the critical review of our protocol.

\section{Author affiliations}

1. D'Or Institute for Research and Education (IDOR), Rio de Janeiro, Brazil.

2. Division of Orthopedic Surgery, Hamad Medical Corporation, Doha, Qatar

3. Consultant Orthopedic \& Trauma Surgeon, Subspecialist in Orthopaedic Oncology and arthroplasty, PPCR certified (Harvard), Department of Orthopedic Surgery, (AFH), Muscat, Sultanate of Oman

4. Pontificia Universidad Católica Madre y Maestra, Santiago De Los Caballeros, República Dominicana.

5. University of Alabama, Tuscaloosa, Alabama, USA.

6. Pontifícia Universidade Católica do Paraná, Londrina, Brazil.

7. Charité Medical University of Berlin (Campus Virchow Klinikum), Berlin, Germany

8. Hospital Garrahan, Emergency Department, Universidad de Buenos Aires, Buenos Aires, Argentina

9. Centro de Investigación de Medicina de Precisión, Universidad San Martin de Porres, Lima, Peru.

10. University Hospital Getúlio Vargas. Amazonas Federal University. Neurosurgery Department. Manaus, Amazonas, Brazil.

11. Pontificia Universidad Católica Madre y Maestra, Santiago De Los Caballeros, República Dominicana.

12. Harvard Medical School, Boston, Massachusetts, USA. Brigham and Women's Hospital, Boston, Massachusetts, USA

13. COVANCE Inc, Clinical Operations Department, Bogota DC, Colombia.

14. Postgraduate Program in Anesthesiology, Surgical Sciences, and Perioperative Medicine, University of São Paulo Faculty of Medicine (FMUSP), São Paulo, Brazil

15. Pontificia Universidad Católica Madre y Maestra, Santiago De Los Caballeros, República Dominicana.

16. HCor Research Institute (IP-HCor), Hospital do Coração, São Paulo, Brazil; Graduate Program in Health Sciences (Cardiology), Instituto de Cardiologia/Fundação Universitária de Cardiologia do Rio Grande do Sul (IC/FUC), Porto Alegre, Brazil.

17. Neurologic Clinic and Policlinic, Department of Medicine, University Hospital Basel, Basel, Switzerland.

18. Universidade Salvador (UNIFACS), Salvador, Brazil

19. Brazilian Cancer Control Institute (IBCC), São Paulo, Brazil.

20. Sírio Libanes Hospital- Brasília Unity; Brasília Hospital, Brasília, Brazil

21. Centro de Estudios Clínicos, Instituto de Ciencias e Innovación en Medicina, Facultad de Medicina Clínica Alemana Universidad del Desarrollo, Santiago, Chile
22. King Abdullah International Medical Research Center/ King Saud bin Abdul Aziz University for Health Sciences, National Guard Health Affairs, Jeddah, Saudi Arabia

23. Assistant Professor Neurology and Cerebrovascular Diseases, The Max Rady Faculty of Health Sciences, University of Manitoba, Winnipeg, Canada.

24. Professor Doctor at the School of Veterinary Medicine and Animal Science (FMVZ), University of São Paulo (USP), São Paulo, Brazil.

25. Centro de Atención y Aislamiento temporal COVID-19, Villa Panamericana, Corporación Futura Farms, Lima, Peru 26. Division of Infectious Diseases, Department of Pediatrics, McGovern Medical School, University of Texas Health Sciences Center at Houston, Houston, TX USA

27. Division of Liver Transplant and HPB Surgery, Department of Surgery, Hamad Medical Corporation, Doha, Qatar

\section{Conflicts of interest and financial disclosure}

The authors have no financial or personal conflicts of interest related to this protocol. The final version of this manuscript was approved by all authors.

\section{REFERENCES}

American Psychiatric Association (2013). American Psychiatric Association: Diagnostic and Statistical Manual of Mental Disorders. 5th ed. Arlington.

Chan, M. T., Cheng, B. C., Lee, T. M., Gin, T., \& CODA Trial Group. (2013). BISguided anesthesia decreases postoperative delirium and cognitive decline. Journal of neurosurgical anesthesiology, 25(1), 33-42.

Evered, L., Silbert, B., Knopman, D.S., Scott, D.A., DeKosky, S.T., Rasmussen, L.S., Oh, E.S., ... \& Eckenhoff, R.G. (2018). Nomenclature Consensus Working Group. Recommendations for the nomenclature of cognitive change associated with anaesthesia and surgery-2018. Br J Anaesth, 121(5),1005-1012

Fabbri, R.M., Moreira, M.A., Garrido, R., Almeida, O.P. (2001). Validity and reliability of the Portuguese version of the Confusion Assessment Method (CAM) for the detection of delirium in the elderly. Arq Neuropsiquiatr, 59(2-A), 175-179.

Gleason, L.J., Schmitt, E.M., Kosar, C.M., Tabloski, P., Saczynski, J.S., Robinson, T., Cooper, Z., ... \& Inouye, S.K. (2015). Effect of Delirium and Other Major Complications After Elective Surgery in Older Adults. JAMA Surgery, 150(12), 1134-1140.

Gonzalvo, C. M., de Wit, H. A., van Oijen, B. P., Deben, D. S., Hurkens, K. P., Mulder, W. J., ... \& van der Kuy, P. H. M. (2017). Validation of an automated delirium prediction model (DElirium MOdel (DEMO)): an observational study. BMJ open, 7(11), e016654.

Harman, N. L., Conroy, E. J., Lewis, S. C., Murray, G., Norrie, J., Sydes, M. R. ... \& Campbell, M. K. (2015). Exploring the role and function of trial steering committees: results of an expert panel meeting. Trials, 16(1), 597.

Hshieh, T. T., Yue, J., Oh, E., Puelle, M., Dowal, S., Travison, T., \& Inouye, S. K. (2015). Effectiveness of multicomponent nonpharmacological delirium interventions: a meta-analysis. JAMA internal medicine, 175(4), 512-520.

Inouye, S. K., van Dyck, C. H., Alessi, C. A., Balkin, S., Siegal, A. P., \& Horwitz, R. I. (1990). Clarifying confusion: the confusion assessment method. A new method for detection of delirium. Annals of internal medicine, 113(12), 941-948.

Knol W., Keijsers CJ., Jansen PA., Belitser, SV., Schobben, AFAM., Egberts, ACG., Van Marum, RJ. (2009). Validity and reliability of the Simpson- 
Angus Scale (SAS) in drug induced parkinsonism in the elderly. Int J Geriatr Psychiatr, 24(2),183-189.

Larsen, K. A., Kelly, S. E., Stern, T. A., Bode, R. H., Price, L. L., Hunter, D. J., ... \& Cotter, J. J. (2010). Administration of olanzapine to prevent postoperative delirium in elderly joint-replacement patients: a randomized, controlled trial. Psychosomatics, 51(5), 409-418.

Leslie, D. L., Marcantonia, E. R., Zhang, Y., Leo-Summers, L., Inouye, S. K. (2008) One-Year Health Care Costs Associated with Delirium in the Elderly. Archives of Internal Medicine. 168(1): 27-32.

Lewis SR, Pritchard MW, Fawcett $\sqcup$, Punjasawadwong Y. (2019) Bispectral index for improving intraoperative awareness and early postoperative recovery in adults. Cochrane Database of Systematic Reviews 26, 9(9), CD003843.

Litaker, D., Locala, J., Franco, K., Bronson, D. L., \& Tannous, Z. (2001). Preoperative risk factors for postoperative delirium. General hospital psychiatry, 23(2), 84-89.

MacLullich, A. M., Ferguson, K. J., Miller, T., de Rooij, S. E., \& Cunningham, C. (2008). Unravelling the pathophysiology of delirium: a focus on the role of aberrant stress responses. Journal of psychosomatic research, 65(3), 229-238.

Maldonado, J,R. (2008) Delirium in the Acute Care Setting: Characteristics, Diagnosis and Treatment. Critical Care Clinics, 24(4), 657-722.

Markowitz, J. D. \& Meera, N. (2008) Delirium and antipsychotics: a systematic review of epidemiology and somatic treatment options. Psychiatry (Edgmont) 5(10), 29-36.

Nasreddine ZS, Phillips NA, Bédirian V, Charbonneau S, Whitehead V, Collin I, Cummings JL, Chertkow H. (2005). The Montreal Cognitive Assessment, MoCA: a brief screening tool for mild cognitive impairment. J Am Geriatr Soc, 53(4), 695-9.

Rivière, J., van der Mast, R. C., Vandenberghe, J., \& Van Den Eede, F. (2019). Efficacy and tolerability of atypical antipsychotics in the treatment of delirium: a systematic review of the literature. Psychosomatics, 60(1), 18-26.

Siddiqi, N., Harrison, J. K., Clegg, A., Teale, E. A., Young, J., Taylor, J., \& Simpkins, S. A. (2016). Interventions for preventing delirium in hospitalized non-ICU patients. Cochrane Database of Systematic Reviews, (3)

Skrobik, Y. K., Bergeron, N., Dumont, M., \& Gottfried, S. B. (2004). Olanzapine vs haloperidol: treating delirium in a critical care setting. Intensive care medicine, 30(3), 444-449.

Trotti A., Colevas AD., Setser A., Rusch V., Jaques D., Budach, V., Langer, C., ... Rubin, P. (2003). CTCAE v3.0: development of a comprehensive grading system for the adverse effects of cancer treatment. Semin Radiat Oncol, 13(3),176-181.

Whitlock, E. L., Vannucci, A., \& Avidan, M. S. (2011). Postoperative delirium. Minerva anestesiologica, 77(4), 448.

Witlox, J., Eurelings, L. S., de Jonghe, J. F. M., Kalisvaart, K. J., Eikelenboom, P., van Gool, W. A., (2010) Delirium in Elderly Patients and the Risk of Postdischarge Mortality, Institutionalization, and Dementia: A Metaanalysis. JAMA, 304(4), 443-451.

Zhou, Y., Li, Y., Wang, K. (2018). Bispectral Index Monitoring During Anesthesia Promotes Early Postoperative Recovery of Cognitive Function and Reduces Acute Delirium in Elderly Patients with Colon Carcinoma: A Prospective Controlled Study using the Attention Network Test. Med Sci Monit, 31(24), 7785-7793.

Copyright: (C) 2021 PPCR. The Principles and Practice of Clinical Research 